\title{
Retrieval of the amplitude and phase of the dipole matrix element by attosecond electron-wave-packet interferometry
}

\author{
Alexis Chacon, ${ }^{1}$ Manfred Lein, ${ }^{2}$ and Camilo Ruiz ${ }^{3}$ \\ ${ }^{1}$ Departamento de Física Aplicada, Universidad de Salamanca, Spain \\ ${ }^{2}$ Institut für Theoretische Physik and Centre for Quantum Engineering and Space-Time Research (QUEST), \\ Leibniz Universität Hannover, Appelstraße 2, D-30167 Hannover, Germany \\ ${ }^{3}$ Centro de Láseres Pulsados, CLPU, Parque Científico, 37185 Villamayor, Salamanca, Spain
}

(Received 28 October 2012; published 13 February 2013)

\begin{abstract}
We extend the ideas of wave-packet interferometry [Remetter et al., Nat. Phys. 2, 323 (2006)] to implement the algorithm of spectral phase interferometry for direct electric-field reconstruction (SPIDER) for characterizing the amplitude and phase of electron wave packets. Single-photon ionization by an attosecond pulse launches an electron wave packet in the continuum. Ionization by a train of two attosecond pulses in the presence of a moderate infrared pulse creates an interferogram in the final photoelectron momentum distribution. From the interferogram, the complex electron wave function can be reconstructed. If the pulses are well characterized, the amplitude and phase of the bound-free dipole matrix element can be reconstructed over a wide energy range. This is demonstrated by application of the retrieval method to momentum distributions obtained by numerical solution of the time-dependent Schrödinger equation. The case of Coulombic potentials requires appropriate treatment of the laser-Coulomb coupled dynamics.
\end{abstract}

DOI: 10.1103/PhysRevA.87.023408

PACS number(s): $32.80 . \mathrm{Rm}, 33.20 . \mathrm{Xx}$

\section{INTRODUCTION}

The advent of new extreme ultraviolet (xuv) sources [1,2] and in particular the attosecond pulses [3] have opened new opportunities for imaging atomic and molecular systems with unprecedented temporal resolution [4,5]. Attosecond pulses ( 1 as $=10^{-18} \mathrm{~s}$ ) have a broadband spectrum in the xuv and can ionize an atom or a molecule by single-photon absorption leading to emission of an electron wave packet (EWP). The EWP contains information about both the attosecond pulse [6] and the atomic or molecular system [4,7]. The spectrum of the attosecond pulses and the bound-free transition dipole matrix element are mapped onto the amplitude and bandwidth of the electron spectrum.

The dipole matrix element contains structural information $[8,9]$ about the initial state. Ionization by attosecond pulses and measurement of the electron spectra provide access to the absolute value of the dipole transition matrix element over a broad range of energies. But the phase of this quantity remains largely unknown and hard to measure. For a complete characterization of the system, the knowledge of this phase is essential. It also determines the temporal structure of the photoionization through the Wigner time delay [4,10].

In this paper we propose a general technique for the complete characterization of the EWP and the complex dipole matrix element. In contrast to previous work $[6,11]$ we do not focus on the characterization of the attosecond pulse but rather on the characterization of the dipole matrix element. We build upon the previous demonstration of the measurement of the EWP phase difference [7] to propose a general interferometric method for full characterization of the complex EWP and the dipole matrix element. We introduce the quantum spectral phase interferometry for direct electron wave-packet reconstruction (QSPIDER) technique which is a variant of the SPIDER [12,13] scheme, now applied to quantum mechanical wave functions.
The SPIDER technique is an interferometric measurement of the electric field in short laser pulses. It uses a nonrecursive algorithm [14] to extract the phase differences between the different frequency components of the pulse. A frequency shift, $\Delta \omega$, or relative shear is introduced into one delayed copy of the pulse. For a laser pulse, $E(\omega)$, the signal measured in SPIDER can be written as $S(\omega)=\left|E(\omega)+E(\omega+\Delta \omega) e^{-i \omega \tau}\right|^{2}$, where $E(\omega)$ is the electric field of the pulse to be characterized in the frequency domain and $\tau$ is the delay between the two copies [12].

The spectral phase difference $\phi(\omega+\Delta \omega)-\phi(\omega)$ between the two signals is encoded in the position of the fringes in the interferogram. It can be retrieved using a four-step algorithm which consists of a fast Fourier transform (FFT) to the pseudotemporal domain, a filtering operation to isolate the ac component, a removal of the extra phase introduced by the delay $\tau$, and finally an inverse FFT [14-16]. If the shear $\Delta \omega$ is small, the obtained phase difference approximately yields the derivative of the spectral phase.

The SPIDER technique can be applied to characterize attosecond pulses as well. As demonstrated by Quéré and coworkers [6], the spectral phase of such pulses can be obtained by applying SPIDER to the electron wave packets generated by photoionization of an atom by the attosecond pulses, assuming a flat transition dipole. The spectral shear is introduced by the presence of a moderately strong infrared (IR) field. The observable containing the interferogram is the photoelectron momentum distribution. We demonstrate that this technique [6] can be extended to characterize the amplitude and phase of the dipole matrix element of the ionization step.

The organization of the paper is as follows. We first introduce the basic theory of photoionization by attosecond pulses in the presence of an IR laser field. We derive the interferometric signal with all involved phases. In Sec. III, we describe how to implement QSPIDER, i.e., how to retrieve the phases from the photoelectron momentum distribution. In 
Sec. IV, we present examples using momentum distributions calculated within the strong-field approximation (SFA). The subsequent sections present numerical simulations based on the time-dependent Schrödinger equation (TDSE). We report results for both short-range and long-range Coulombic potentials. We show that special care is needed in the latter case due to the laser-Coulomb coupling in the electron dynamics.

\section{PHOTOIONIZATION IN THE PRESENCE OF AN INFRARED FIELD}

In this section we describe how the information about the transition dipole matrix element is encoded into the electron interferogram.

The ionization by a single attosecond pulse in the presence of a moderate IR laser pulse can be described by considering the bound-free transition amplitude $a_{\mathbf{p}}=\left\langle\mathbf{p}\left|\hat{U}\left(t_{f}, t_{0}\right)\right| \Psi_{0}\right\rangle$ from the initial state $\left|\Psi_{0}\right\rangle$ at time $t_{0}$ before the pulses to a final continuum state $|\mathbf{p}\rangle$ at the detection time $t_{f}$ evolving according to the operator $\hat{U}\left(t_{f}, t_{0}\right)$. An approximate treatment of this transition is obtained by using perturbation theory for the xuv-induced ionization and the SFA $[17,18]$ for the coupling to the IR field (atomic units are used in this paper):

$$
\begin{aligned}
a_{\mathbf{p}}(\tau)= & i \int_{t_{0}}^{t_{f}} d t^{\prime} e^{i\left[I_{p}\left(t^{\prime}-t_{0}\right)-\frac{1}{2} \int_{t^{\prime}}^{t_{f}}\left[\left(\mathbf{p}+\mathbf{A}_{L}\left(t^{\prime \prime}\right)\right]^{2} d t^{\prime \prime} \mathbf{I}\right.\right.} \\
& \times \mathbf{E}_{X}\left(t^{\prime}-\tau\right) \cdot \mathbf{d}\left[\mathbf{p}+\mathbf{A}_{L}\left(t^{\prime}\right)\right] .
\end{aligned}
$$

Here $I_{p}$ is the ionization potential and $\mathbf{A}_{L}(t)=-\int^{t} \mathbf{E}_{L}\left(t^{\prime}\right) d t^{\prime}$ is commonly referred to as the vector potential of the IR field $\mathbf{E}_{L}(t)$. We consider a linearly polarized IR pulse and xuv pulses linearly polarized along the same axis. The xuv field is $\mathbf{E}_{X}(t)=\mathbf{e}_{x} \tilde{E}_{0, X}(t) \operatorname{Re}\left[e^{-i \omega_{X} t+i \varphi_{X}(t)}\right]$ with envelope $\tilde{E}_{0, X}(t)$ and the central frequency $\omega_{X}$. Here $\varphi_{X}(t)$ is the phase of the xuv pulse which may contain, for example, a chirp. In the notation of Eq. (1), the xuv pulse is centered at $\tau$.

In the SFA approach, the final momentum $|\mathbf{p}\rangle$ is approximated by a plane wave and the dipole matrix element is defined as $\mathbf{d}\left[\mathbf{p}+\mathbf{A}_{L}(t)\right]=-\left\langle\mathbf{p}+\mathbf{A}_{L}(t)|\mathbf{r}| \Psi_{0}\right\rangle$. According to Eq. (1), the ionization is mediated by the xuv pulse only, while the IR laser pulse contributes to the phase of the EWP and changes the momentum from $\mathbf{p}+\mathbf{A}_{L}(t)$ at the time of ionization to $\mathbf{p}$ at the final time.

A more convenient formulation can be used when the attosecond pulse is much shorter than the period of the IR pulse. The final momentum-space wave function is the product of an amplitude and a phase factor. For an attosecond pulse centered at $\tau_{1}$ we write

$$
a_{\mathbf{p}}\left(\tau_{1}\right)=A\left(\mathbf{p}, \tau_{1}\right) e^{i \phi\left(\mathbf{p}, \tau_{1}, t_{f}\right)},
$$

with

$$
\begin{aligned}
A\left(\mathbf{p}, \tau_{1}\right)= & \frac{1}{2}\left|d\left[\mathbf{p}+\mathbf{A}_{L}\left(\tau_{1}\right)\right]\right|\left|E_{0, X}\left(\omega_{\mathbf{p}}\right)\right|, \\
\phi\left(\mathbf{p}, \tau_{1}, t_{f}\right)= & \phi_{d\left[\mathbf{p}+\mathbf{A}_{L}\left(\tau_{1}\right)\right]}+I_{p}\left(\tau_{1}-t_{0}\right) \\
& -\frac{1}{2} \int_{\tau_{1}}^{t_{f}}\left[\mathbf{p}+\mathbf{A}_{L}\left(t^{\prime \prime}\right)\right]^{2} d t^{\prime \prime} \\
& +\phi_{X}\left(\omega_{\mathbf{p}}\right)+\phi_{\mathrm{LIC}}\left(\mathbf{p}, \tau_{1}\right)+\frac{\pi}{2} .
\end{aligned}
$$

Here $d(\mathbf{p})=\mathbf{d}(\mathbf{p}) \cdot \mathbf{e}_{x}$ is the component of the dipole along the polarization axis. The expression $E_{0, X}\left(\omega_{\mathbf{p}}\right)=$ $\left|E_{0, X}\left(\omega_{\mathbf{p}}\right)\right| e^{i \phi_{X}\left(\omega_{\mathbf{p}}\right)}$ is the Fourier transform of $\tilde{E}_{0, X}(t) e^{i \varphi_{X}(t)}$ as a function of $\omega_{\mathbf{p}}$ defined as $\omega_{\mathbf{p}}=\frac{1}{2}\left[\mathbf{p}+\mathbf{A}_{L}\left(\tau_{1}\right)\right]^{2}-\frac{p_{0}^{2}}{2}$ and $\frac{p_{0}^{2}}{2}=\omega_{X}-I_{p}$. To obtain Eq. (3), we have expanded the phase integral in the exponent of Eq. (1) to second order in $t^{\prime}$ around $\tau_{1}$, with $\mathbf{A}_{L}\left(t^{\prime}\right) \approx \mathbf{A}_{L}\left(\tau_{1}\right)-\mathbf{E}_{L}\left(\tau_{1}\right)\left(t^{\prime}-\tau_{1}\right)$. We further neglect the changes in the dipole due to the expansion and approximate $\mathbf{d}\left[\mathbf{p}+\mathbf{A}_{L}\left(t^{\prime}\right)\right] \approx \mathbf{d}\left[\mathbf{p}+\mathbf{A}_{L}\left(\tau_{1}\right)\right]$.

In Eq. (4) $\phi_{X}\left(\omega_{\mathbf{p}}\right)$ is the spectral phase of the attosecond pulse which may, for example, contain an intrinsic chirp [19] and the xuv carrier envelope phase (CEP) as well. The phase $\phi_{\text {LIC }}\left(\mathbf{p}, \tau_{1}\right)$ describes the laser-induced chirp (LIC) of the EWP caused by the variations of the IR field around the time of ionization. Assuming a Gaussian pulse shape $\tilde{E}_{0, X}(t) \sim e^{-\alpha t^{2}}$, this phase is given by

$$
\begin{aligned}
\phi_{\text {LIC }}\left(\mathbf{p}, \tau_{1}\right)= & -\frac{1}{2} \arctan \left[\frac{\Gamma\left(\mathbf{p}, \tau_{1}\right)}{2 \alpha}\right]+\frac{2 \Gamma\left(\mathbf{p}, \tau_{1}\right)}{(4 \alpha)^{2}+\left(2 \Gamma\left(\mathbf{p}, \tau_{1}\right)\right)^{2}} \\
& \times\left[\frac{1}{2}\left[\mathbf{p}+\mathbf{A}_{L}\left(\tau_{1}\right)\right]^{2}-\frac{p_{0}^{2}}{2}\right]^{2}
\end{aligned}
$$

with $\Gamma(\mathbf{p})=\left[\mathbf{p}+\mathbf{A}_{L}\left(\tau_{1}\right)\right] \cdot \mathbf{E}_{L}\left(\tau_{1}\right)$. The LIC phase is responsible for the different widths of the EWP in the position space and is a measure of the attosecond pulse duration with respect to the variations of the vector potential. For short attosecond pulses $\left(<200\right.$ as) and modest intensity $\left(I<10^{13} \mathrm{~W} / \mathrm{cm}^{2}\right)$ the effects of the LIC phase are negligible. This phase depends on the value of the electric field at ionization time $\tau_{1}$ and is zero if $\mathbf{E}_{L}\left(\tau_{1}\right)=0$. A similar phase exists in the case of the optical SPIDER when an insufficiently chirped ancilla is used [20]. This phase will become relevant for streaking and interferometric measurements as it can become larger than the phase of the dipole.

In order to implement the QSPIDER technique, two delayed copies of the EWP with a relative shear between them are needed to construct an interferogram. For this we use an attosecond pulse train (APT) with two pulses centered at $\tau_{1}$ and $\tau_{2}$ in the presence of the weak IR laser pulse. The two pulses generate two EWPs which are delayed relative to each other by the separation of the pulses in the train [6,21]. The weak IR laser pulse streaks each of the EWPs by the value of the vector potential at the ionization time [11] to produce a relative streaking, $\Delta \mathbf{A}_{L}=\mathbf{A}_{L}\left(\tau_{2}\right)-\mathbf{A}_{L}\left(\tau_{1}\right)$, between the two copies. The streaked and delayed copies produce an interferogram in the final momentum distribution which is conceptually equivalent to the interferogram of the SPIDER technique. According to the SFA, the interferogram is

$$
\begin{aligned}
\left|a_{\mathbf{p}}\left(\tau_{1}, \tau_{2}\right)\right|^{2}= & \left|A_{1}\left(\mathbf{p}, \tau_{1}\right) e^{-i \phi\left(\mathbf{p}, \tau_{1}, t_{f}\right)}+A_{2}\left(\mathbf{p}, \tau_{2}\right) e^{-i \phi\left(\mathbf{p}, \tau_{2}, t_{f}\right)}\right|^{2} \\
= & A_{1}\left(\mathbf{p}, \tau_{1}\right)^{2}+A_{2}\left(\mathbf{p}, \tau_{2}\right)^{2} \\
& +2 A_{1}\left(\mathbf{p}, \tau_{1}\right) A_{2}\left(\mathbf{p}, \tau_{2}\right) \cos \left(\Delta \phi_{21}\right) .
\end{aligned}
$$

Here, $A_{1}\left(\mathbf{p}, \tau_{1}\right)$ and $A_{2}\left(\mathbf{p}, \tau_{2}\right)$ are the (real) amplitudes for each of the EWPs. The interference term in Eq. (6) is governed by the relative phase

$$
\begin{aligned}
\Delta \phi_{21}= & \Delta \phi_{d}\left(\mathbf{p}, \tau_{1}, \tau_{2}\right)+\frac{1}{2} \int_{\tau_{1}}^{\tau_{2}} d t^{\prime \prime}\left[\mathbf{p}+\mathbf{A}_{L}\left(t^{\prime \prime}\right)\right]^{2} \\
& +I_{p} \Delta \tau+\Delta \phi_{X}\left(\mathbf{p}, \tau_{1}, \tau_{2}\right)+\Delta \phi_{\mathrm{LIC}}\left(\mathbf{p}, \tau_{1}, \tau_{2}\right) .
\end{aligned}
$$


Here, $\Delta \tau=\tau_{2}-\tau_{1}$ is the delay between the attosecond pulses and $\Delta \phi_{d}\left(\mathbf{p}, \tau_{1}, \tau_{2}\right)=\phi_{d\left[\mathbf{p}+\mathbf{A}_{L}\left(\tau_{2}\right)\right]}-\phi_{d\left[\mathbf{p}+\mathbf{A}_{L}\left(\tau_{1}\right)\right]}$ is the phase difference of the dipole matrix element.

In this approximation the interference depends on five terms. The first one, $\Delta \phi_{d}\left(\mathbf{p}, \tau_{1}, \tau_{2}\right)$, is related to the phase of the dipole transition matrix element. The second term is the Volkov phase related to the different time spent in the continuum by the two EWPs. The third term is the accumulated phase difference in the initial state before ionization, which we call the atomic phase. The two last terms are related to the spectral phases of the attosecond pulses, which also contain the carrier envelope phase difference, and to the LIC.

The interference between the two streaked EWPs depends on the emission direction. Assuming a small collection angle around the polarization direction, we can restrict ourselves to one-dimensional modeling; i.e., we focus on electrons emitted along the polarization axis.

The theory outlined above is suitable only for systems that are well described by the SFA, i.e., potentials without a Coulomb tail. For Coulombic potentials, the separation of the electron dynamics into the two steps of ionization and interaction with the laser field is problematic because the electron interacts with the laser field and the long-range potential at the same time. The long-range character is also reflected in the field-free continuum states that are needed in the transition dipole matrix elements. For the potential $V(x)$ with asymptotic behavior $-Z /|x|$, the positive-momentum component of the continuum state $\Phi_{k}(x)$ for the asymptotic momentum $k>0$ behaves as

$$
\Phi_{k}(x) \approx \exp [i k x+i Z \ln (2 k x) / k], \text { for } x \rightarrow \infty .
$$

For the Coulomb-laser coupled dynamics we follow the eikonal approach by Ivanov and coworkers [22]. For the purpose of calculating the time evolution of the eikonal state, we assume an electron trajectory starting at a distance $x_{0}$ and moving outwards [23]. One finds that the position representation of the continuum state after evolving from the time of ionization $\tau$ to the final time $t_{f}$ is

$$
\begin{aligned}
\left\langle x\left|\hat{U}\left(t_{f}, \tau\right)\right| p+A_{L}(\tau)\right\rangle \\
=e^{i p x} e^{-i \int_{\tau}^{t_{f}} d t\left[p+A_{L}(t)\right]^{2} / 2} e^{-i \int_{\tau}^{t_{f}} d t V\left[x_{0}+p(t-\tau)+\int_{\tau}^{t} d t^{\prime} A_{L}\left(t^{\prime}\right)\right]} \\
\quad \times e^{-\frac{i}{p+A_{L}(\tau)} \int_{\mathrm{ref}}^{x_{0}} d l V(l)}
\end{aligned}
$$

where $|p\rangle$ denotes the eikonal continuum state with asymptotic momentum $p$ and $\hat{U}\left(t_{f}, \tau\right)$ is the approximate time evolution operator. Since we are primarily interested in the phase correction due to the laser-Coulomb coupling, we have omitted the amplitude correction of the wave function in Eq. (9). In the following, we assume that the initial position is $x_{0}=1 /[2(p+$ $A(\tau)$ ] [23] and furthermore we set $x_{0}=x_{\text {ref }}$ in accordance with the boundary condition of Eq. (8). Thus, the last phase factor in Eq. (9) is equal to 1. After the substitution $s=x_{0}+p(t-\tau)$ and inserting the Coulomb potential $V(x)=-Z /|x|$ we obtain for positive $x$ that

$$
\begin{aligned}
\left\langle x\left|\hat{U}\left(t_{f}, \tau\right)\right| p+A_{L}(\tau)\right\rangle= & e^{i p x} e^{-i \int_{\tau}^{t_{f}} d t\left[p+A_{L}(t)\right]^{2} / 2} \\
& \times e^{\frac{i Z}{p} \int_{x_{0}}^{x_{f}} d s\left[s+\int_{\tau}^{\tau+\left(s-x_{0}\right) / p} d t^{\prime} A_{L}\left(t^{\prime}\right)\right]^{-1}},
\end{aligned}
$$

where $x_{f}=x_{0}+p\left(t_{f}-\tau\right)$.
Finally, we consider two EWPs with the same asymptotic momentum $p$, but launched at two different times, $\tau_{1}$ and $\tau_{2}$. In the limit $t_{f} \rightarrow \infty$, we find that the two wave functions differ by a relative phase

$$
\begin{aligned}
\Delta \phi_{\mathrm{VC}}\left(p, \tau_{1}, \tau_{2}\right)= & \frac{1}{2} \int_{\tau_{1}}^{\tau_{2}} d t\left[p+A_{L}(t)\right]^{2} \\
& +\lim _{x_{f} \rightarrow \infty}\left(\phi_{\mathrm{C}}\left(p, \tau_{2}\right)-\phi_{\mathrm{C}}\left(p, \tau_{1}\right)\right), \\
\phi_{\mathrm{C}}\left(p, \tau_{j}\right)= & \frac{Z}{p} \int_{x_{0, j}}^{x_{f}} \frac{d s}{s+\int_{\tau_{j}}^{\tau_{j}+\left(s-x_{0, j}\right) / p} d t^{\prime} A_{L}\left(t^{\prime}\right)},
\end{aligned}
$$

where $x_{0, j}=1 /\left[2\left(p+A_{L}\left(\tau_{j}\right)\right]\right.$ for $j=1,2$. We conclude that in the case of Coulombic potentials, Eq. (7) is replaced by the Coulomb-corrected phase difference

$$
\Delta \phi_{21}=\Delta \phi_{d}+\Delta \phi_{\mathrm{VC}}+I_{p} \Delta \tau+\Delta \phi_{X}+\Delta \phi_{\mathrm{LIC}},
$$

where we have omitted the arguments for notational simplicity.

\section{IMPLEMENTATION OF QSPIDER}

QSPIDER is a quantum implementation of the SPIDER technique. The interference of two delayed EWPs leads to an interferogram in the electron momentum distribution, where the relevant phase difference $\Delta \phi_{21}$ is given by Eq. (7) or (12).

The technique first measures the phase difference $\Delta \phi_{21}$. For metrology applications as suggested by Quéré et al. [6], the spectral phase of the xuv pulse can be recovered to reconstruct the temporal shape of the xuv pulse. This is possible if $\Delta \phi_{d}$ and the Coulomb corrections are known. In Ref. [6] it was assumed that these phases vanish, which is not fulfilled for a real atom. In contrast, our aim is to retrieve the dipole matrix element, assuming that the xuv field is well known. This also requires the knowledge of quantities such as the relative shear and the delay between the pulses. All of these quantities can be measured and used as input information for QSPIDER.

The scheme for measuring $\Delta \phi_{21}$ is depicted in Fig. 1 . Figure 1(c) shows a typical momentum spectrum as produced by the applied fields shown in Fig. 1(a). This interferogram carries information on the amplitude and phase of the EWPs. The QSPIDER technique for amplitude and phase retrieval is useful only for a limited range of delays and relative shears between the copies, similar to SPIDER [12,13]. These restrictions come from the conditions under which the retrieval algorithm can be applied.

Once the interferogram is available we can apply the SPIDER retrieval algorithm which consists of four steps. First we compute the FFT of the interferogram, transforming from momentum space to pseudoposition space. The resulting signal is depicted in Fig. 1(d). It consists of one central peak, the dc component, and two lateral signals, the ac components. By placing a soft mask, the central dc part can be isolated to obtain the square amplitude of the EWP $\left|A\left(\mathbf{p}, \tau_{1}\right)\right|^{2}$. This simple procedure allows us to retrieve the amplitude of the EWP as if only one attosecond was used. To extract the phase difference $\Delta \phi_{21}$ it is necessary to use a soft mask [dotted red line in Fig. 1(d)] to isolate the right ac term.

For a correct retrieval of this phase the $\mathrm{dc}$ and ac components need to be well separated. This can be controlled by adjusting the delay $\Delta \tau$ between the attosecond pulses, the 

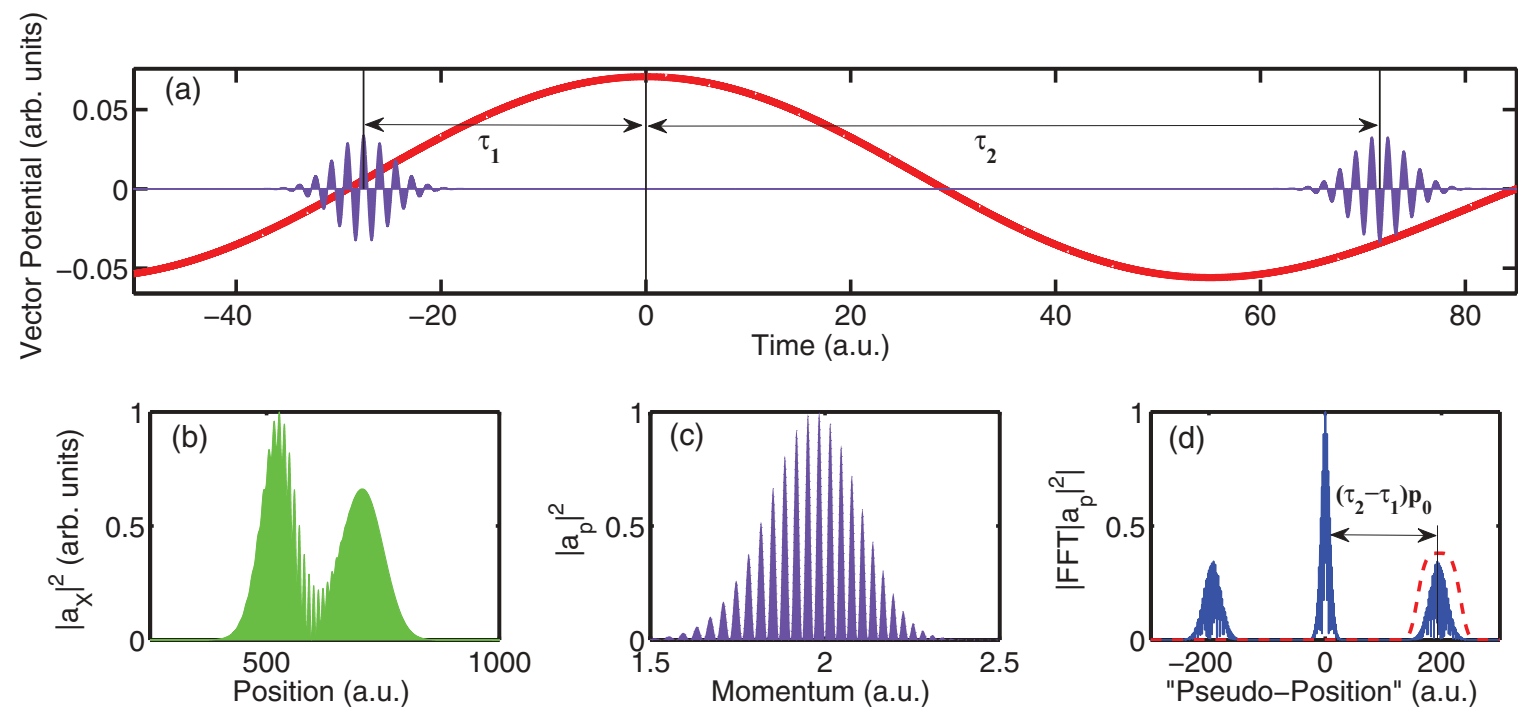

FIG. 1. (Color online) General idea of the QSPIDER technique. (a) Two attosecond pulses (violet area) in the presence of a weak vector potential of the IR laser (red solid line) produce two identical EWPs with different central momentum due to the streaking of the IR field. Panel (b) shows two EWPs some time after the absorption in position space (green area). (c) The momentum distribution of these EWPs is an interferogram (violet area) containing information about the amplitude and phase difference of the dipole matrix elements. (d) The FFT of the momentum distribution consists of a de central component and two ac components (blue line), which is spaced by $\left(\tau_{2}-\tau_{1}\right) p_{0}$ with respect to the dc. After isolating the right ac (red dashed line), the phase difference can be recovered.

central momentum of the EWP, and the spectral width of the xuv pulse. For the attosecond pulses considered here, the best retrievals are obtained for delays around $\Delta \tau \sim T_{\mathrm{IR}}$, the period of the laser pulse. Smaller delays $\Delta \tau$ lead to overlap between the ac and dc components, which are separated by $p_{0} \Delta \tau$, thus disturbing the retrieval algorithm. Attosecond pulses separated by one IR laser cycle have been demonstrated in high-order harmonic generation (HHG) experiments with two colors $(\omega, 2 \omega)$ [24]. Furthermore, the delay of two attosecond pulses can be controlled by means of a segmented mirror [25]. After isolating the ac component, we compute the inverse FFT to obtain the phase difference $\Delta \phi_{21}$.

In analogy to the optical SPIDER technique, we can remove the known phases in Eq. (7) or (12) to extract the phase difference of the complex dipole transition matrix $\Delta \phi_{d}\left(\mathbf{p}, \tau_{1}, \tau_{2}\right)$. In principle, the phase difference can be obtained regardless of the value of the relative streaking between the two copies but if the relative streaking $\Delta A_{L}=A_{L}\left(\tau_{2}\right)-A_{L}\left(\tau_{1}\right)$ is sufficiently small the phase difference gives the derivative of the phase:

$$
\Delta \phi_{d}\left(\mathbf{p}, \tau_{1}, \tau_{2}\right) \approx \frac{\partial \phi_{d}}{\partial p} \Delta A_{L} .
$$

Finally, integration with respect to momentum yields the phase of the dipole matrix element.

The optical SPIDER technique is not able to retrieve the linear spectral phase term, which determines the absolute timing of the pulse, nor the absolute phase [13].

In QSPIDER, the situation is somewhat different: the linear term of the momentum-dependent dipole phase can be retrieved since all other contributions in Eq. (7) can be computed, but the absolute dipole phase cannot be retrieved. Therefore, when integrating the retrieved phase derivative to obtain $\phi_{d}$, an arbitrary integration constant may be added. The removal of the Volkov phase,

$$
\begin{aligned}
\frac{1}{2} \int_{\tau_{1}}^{\tau_{2}} d t^{\prime \prime}\left[\mathbf{p}+\mathbf{A}_{L}\left(t^{\prime \prime}\right)\right]^{2}= & \frac{p^{2}}{2} \Delta \tau+\mathbf{p} \cdot \int_{\tau_{1}}^{\tau_{2}} \mathbf{A}_{L}\left(t^{\prime \prime}\right) d t^{\prime \prime} \\
& +\frac{1}{2} \int_{\tau_{1}}^{\tau_{2}} \mathbf{A}_{L}^{2}\left(t^{\prime \prime}\right) d t^{\prime \prime}
\end{aligned}
$$

is straightforward if a full characterization of the IR pulse is available. The same holds for the Coulomb-corrected phase of Eq. (11). The delay $\Delta \tau$ can be calibrated by measuring the spacing between fringes in the case of ionization by two attosecond pulses without IR field. The relative shear between the copies could be measured by blocking alternatively one of the attosecond pulses while leaving the IR laser pulse; this would result in the momentum distribution streaked by the value of the vector potentials $A_{L}\left(\tau_{1}\right)$ and $A_{L}\left(\tau_{2}\right)$ alternatively.

The spectral phase of the xuv pulse $\Delta \phi_{X}$ must be characterized either by some other technique available $[6,11]$ or by an estimation of the intrinsic chirp based on the HHG process producing the APT. When the xuv and IR pulses are known, we can also evaluate the contribution $\Delta \phi_{\text {LIC }}$.

\section{RESULTS WITHIN THE STRONG-FIELD APPROXIMATION}

In this section, we use the SFA theory to calculate the photoelectron momentum distribution for a helium ion. We then apply the QSPIDER method and compare the retrieved dipole matrix element with that used in the SFA calculation. We can assess the quality of the retrieval depending on the different parameters such as the delay between the pulses and the intensity of the streaking field. To evaluate the SFA 
expression we use the differential formulation [26]

$$
\begin{aligned}
\frac{d a_{\mathbf{p}}(\tau)}{d t}= & i \mathbf{E}_{X}(t-\tau) \cdot \mathbf{d}\left[\mathbf{p}+\mathbf{A}_{L}(t)\right] e^{i I_{p}\left(t-t_{0}\right)} \\
& -i \frac{\left[\mathbf{p}+\mathbf{A}_{L}(t)\right]^{2}}{2} a_{\mathbf{p}}(\tau),
\end{aligned}
$$

which can be derived from Eq. (1) by differentiating with respect to the upper limit of the integral $t_{f} \equiv t$. This expression is helpful because it can be integrated with any algorithm for differential equations and does not require the saddle point approximation. The calculation can be parallelized easily using the message passing interface (MPI) [27] paradigm or multiple threads with a graphic card such as CUDA [28].

We use the ground state $\left|\phi_{0}\right\rangle$ of the helium ion with $I_{p}^{(0)}=2.0$ a.u. $(54.4 \mathrm{eV})$ and the first excited state $\left|\phi_{1}\right\rangle$ with $I_{p}^{(1)}=0.5$ a.u. $(13.60 \mathrm{eV})$. For the first case the dipole has a constant phase due to the plane waves used in the SFA matrix elements and the symmetry of the initial state. This helps us to distinguish extra phases occurring in the measurement or in the retrieval algorithm. In the second case the dipole phase is constant except for a phase jump of $\pi$ at a momentum where the amplitude of the dipole is zero.

In the case of the $\left|\phi_{0}\right\rangle$ state we have used the following pulse parameters. The APT consists of two pulses with central frequency $\omega_{X}=2.845$ a.u. $(77.4 \mathrm{eV})$. The pulse envelope is Gaussian for both pulses with FWHM $\sim 170$ as. The intensity of the xuv pulses is $I_{X}=10^{13} \mathrm{~W} / \mathrm{cm}^{2}$. The best delay for the retrieval is close to one IR period and 0.04 a.u. of momentum shear $\Delta A_{L}$. The parameters of the IR pulse used here and in the next sections are wavelength $\lambda_{\mathrm{IR}}=800 \mathrm{~nm}\left(\omega_{\mathrm{IR}}=0.057\right.$ a.u. $)$ and intensity $I_{\mathrm{IR}}=5.0 \times 10^{11} \mathrm{~W} / \mathrm{cm}^{2}$. The time duration of the laser pulse is around FWHM $\sim 4$ fs; the envelope of the field is a Gaussian function.

Figures 2(b) and 2(c) show the retrieved momentum distribution of the EWP for negative and positive momenta compared to the case of the absorption from a single attosecond pulse without an IR field. Also shown is the retrieved derivative of the dipole phase. The results show a very good agreement in the region defined by the spectral momentum width of the EWP. Figures 2(d) and 2(e) show the retrieval of the dipole amplitude and the dipole phase. The amplitude of the dipole
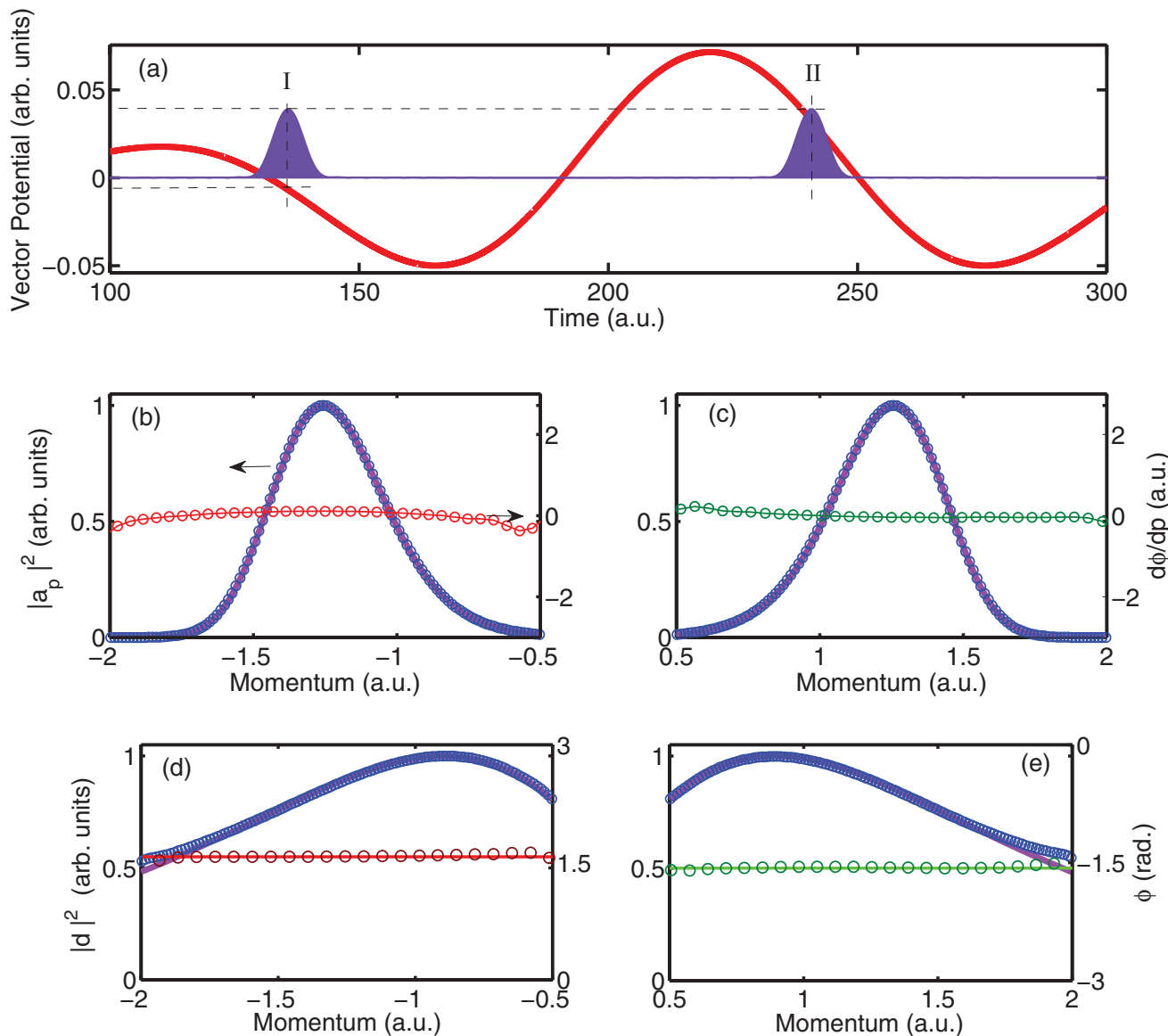

FIG. 2. (Color online) QSPIDER retrieval for the ground state of the $\mathrm{He}^{+}$ion based on SFA momentum distributions. (a) The red solid line shows the vector potential of the IR laser pulse. The violet area is the field envelope of the APT. The retrieved momentum distribution of the EWP (blue circles) compared to the exact momentum distribution (violet solid line) from the interaction with a single attosecond pulse in the absence of the IR field is plotted in panels (b) and (c) for negative and positive momenta. The retrieved derivative of the dipole phase is shown in red and green circles, respectively, in panels (b) and (c). The retrieved squared amplitude (blue circles) and phase (red and green circles) of the dipole matrix element is compared to the exact squared amplitude (violet solid line) and phase (red and green solid lines) in panels (d) and (e). 

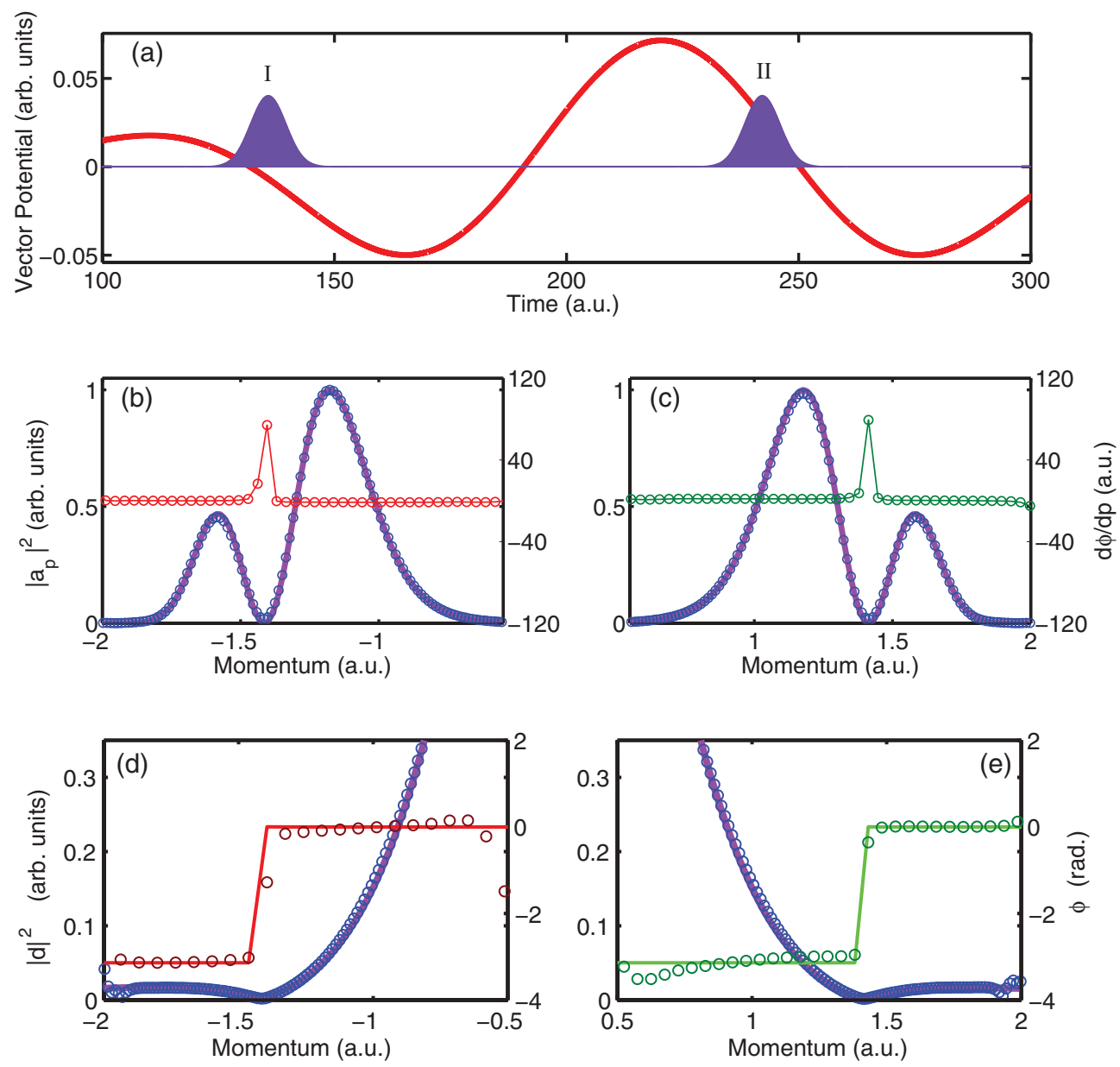

FIG. 3. (Color online) QSPIDER retrieval for the first excited state of the $\mathrm{He}^{+}$ion based on SFA momentum distributions. Lines and symbols are as defined in Fig. 2.

can be found by dividing the retrieved amplitude of the EWP by the xuv amplitude $\left|E_{0, X}\left(\omega_{\mathbf{p}}\right)\right|$ [29]. The agreement between the retrieved amplitude and the exact one is good over all the accessible region. The retrieved dipole phase after integration is constant, which indicates that the algorithm does not add any artificial phase.

In the second case we analyze the first excited state $\left|\phi_{1}\right\rangle$. The dipole matrix element has a node around $p_{x}=1.4 \mathrm{a}$.u. and the phase exhibits a phase jump by $\pi$ at this final momentum. This behavior is similar to that found around the Cooper minimum in photoionization of argon [30]. The sequence of pulses is shown in Fig. 3(a). The APT consists of two pulses with $\omega_{X}=1.605$ a.u. (43.7 eV) and FWHM $\sim 215$ as. The IR field parameters are the same as in the first example.

Figures 3(b) and 3(c) show the retrieved momentum distribution of one EWP compared to the case of the absorption from a single attosecond pulse without an IR field. Also shown is that the retrieved derivative of the phase is in very good agreement with the exact phase derivative. Figures 3(d) and 3(e) show the phase after the integration with the correct jump around $p_{x}=1.4$ a.u. as expected. The agreement between the retrieved and exact dipole amplitudes is good over all the accessible region for negative and positive momentum.

\section{TIME-DEPENDENT SCHRÖDINGER EQUATION (TDSE) FOR A SHORT-RANGE POTENTIAL}

A more realistic description of the phases of the system is the numerical solution of the TDSE. Such calculations include all the couplings in the system exactly. We use the code QFISHBOWL [31] which implements the split-operator technique to obtain the time evolution of the wave function in one, two, and three dimensions. The code uses the parallel FFTW [32], leading to a very fast propagation for any type of input pulse. In this section, we use the ground state ( $I_{p}=0.5$ a.u.) of a one-dimensional model with a short-range potential of the form

$$
V(x)=-\frac{e^{-m \sqrt{a+x^{2}}}}{\sqrt{a+x^{2}}} .
$$

For the calculation of the exact dipole matrix elements, the continuum states need to be computed. These are obtained following a scheme similar to that in Ref. [33] except that we must select the incoming scattering solution in the context of photoionization; i.e., the continuum state is a superposition of incoming scattering wave and outgoing plane wave.

The TDSE momentum distributions are calculated after the end of the APT and the IR pulse. To this end, the TDSE wave 

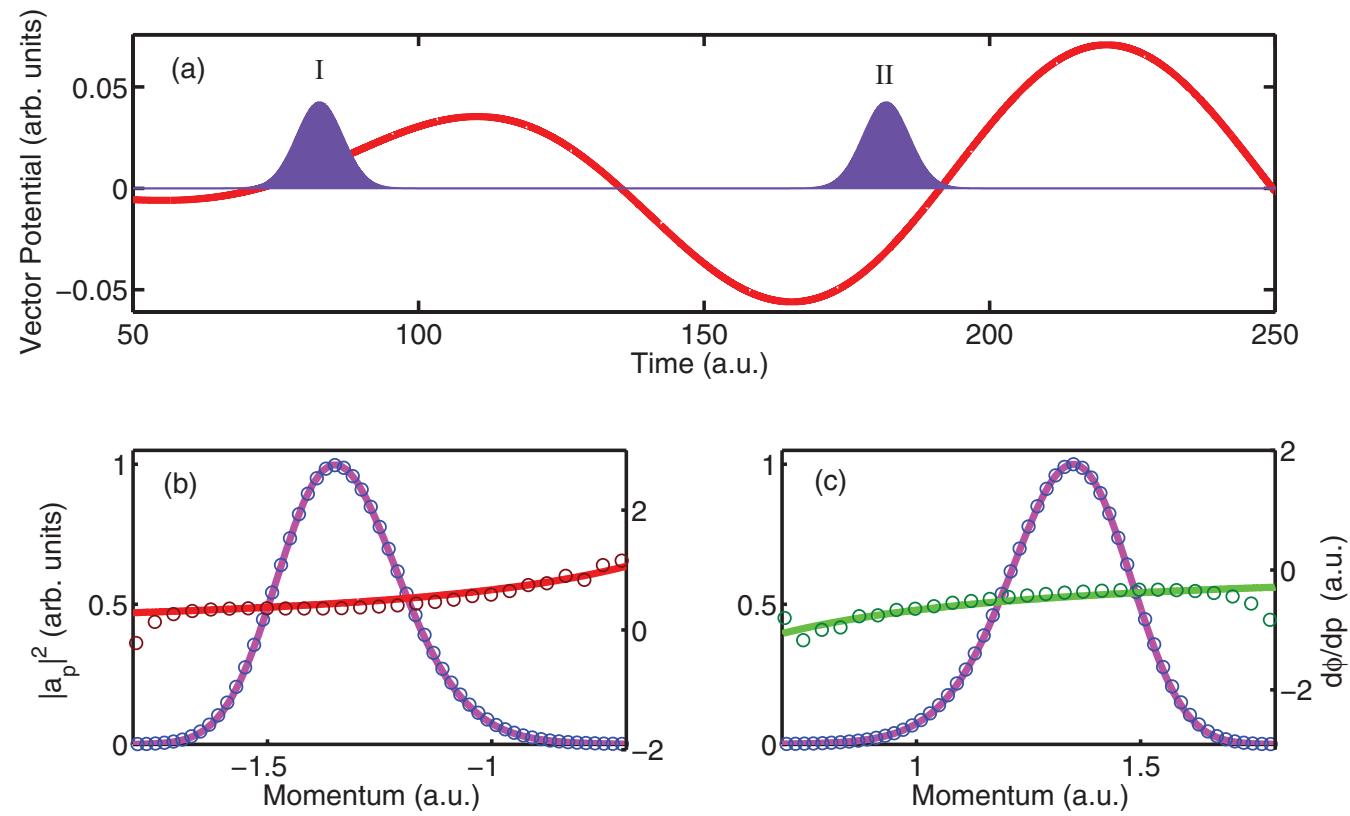

FIG. 4. (Color online) QSPIDER retrieval for the ground state of the short-range potential based on TDSE calculations. Graph (a) shows the IR vector potential (red solid line) and the xuv field envelopes (violet area) used in the TDSE simulation. (b) and (c) QSPIDER retrieval for the EWP amplitude (blue circles) compared to the exact momentum distribution (violet solid line) from the interaction with a single attosecond pulse in absence of the IR field for negative and positive momenta. The retrieved derivative of the dipole phase [red circles in panel (b) and green circles in panel (c)] is compared with the exact dipole phase derivative [red solid line in panel (b) and green solid line in panel (c)].

function is projected on the continuum states. The potential well parameters used in our simulation are $m=a=1 / 2$. The number of grid points is 240000 and the grid spacing is $d x=$ 0.01 a.u. The momentum spacing used for the momentum distribution is $d p=0.001$ a.u.

The APT consists of two pulses with $\omega_{X}=2.0$ a.u. $(54.4 \mathrm{eV})$ and FWHM $\sim 243$ as. The IR field parameters are the same as in the first example, but now the envelope is a sine square function. The delay for the QSPIDER retrieval is chosen close to one IR period, as shown in Fig. 4(a). The momentum shear is $\Delta A_{L}=-0.04$ a.u., i.e., much bigger than the momentum spacing $d p$. Figures 4(b) and 4(c) show the excellent agreement between the retrieved EWP momentum distribution and the case of the absorption from a single attosecond pulse without an IR field. The retrieved derivative of the phase is in very good agreement with the exact phase derivative within the EWP momentum bandwidth. Note that the phase derivative would be equal to zero if plane waves were used in the dipole matrix elements. Thus, our results show that QSPIDER retrieves the linear term in the exact dipole phase quite accurately.

As a general rule, the shear $\Delta A_{L}$ should not be chosen too small, as it was found in the optical SPIDER technique [34]. Otherwise small errors in the measured phase difference $\Delta \phi_{d}$ will lead to large errors in the retrieved phase derivative $\Delta \phi_{d} / \Delta A_{L}$. An estimate of the required shear can be obtained by measuring first the residual phase difference $\Delta \phi_{d}^{(0)}$ for vanishing shear in the presence of the IR field. The shear for QSPIDER should then be chosen such that the measured $\Delta \phi_{d}$ is much larger than $\Delta \phi_{d}^{(0)}$.

\section{TIME-DEPENDENT SCHRÖDINGER EQUATION FOR A COULOMBIC POTENTIAL}

In this section, we consider the ground state of a model helium ion described by the soft-core Coulombic potential:

$$
V(x)=\frac{-2}{\sqrt{a+x^{2}}} .
$$

The photoelectron momentum distribution is obtained from the numerical solution of the TDSE by projection on the exact scattering continuum states. Due to the long-range character of the potential, we require the continuum states to satisfy the boundary condition (8).

The simulation parameters are $d x=0.01$ a.u., 250000 grid points, and soft-core parameter $a=1 / 2$. The attosecond pulse shape is Gaussian for both xuv pulses with FWHM $\sim 170$ as and central frequency $\omega_{X}=4.0$ a.u.

Figures 5(b) and 5(c) show the results. The retrieved derivative of the phase is in good agreement with the exact phase derivative within the EWP momentum bandwidth. In this case both the LIC phase and the Coulomb-laser coupling phase are important and contribute to the overall phase in similar weights. Therefore it is necessary to calculate these contributions with high accuracy. Further, as the derivative of the phase is calculated as division between two quantities, for small values of relative shear $\Delta A_{L}$ we need a great precision for these two phases which are the most sensitive of all considered here. 

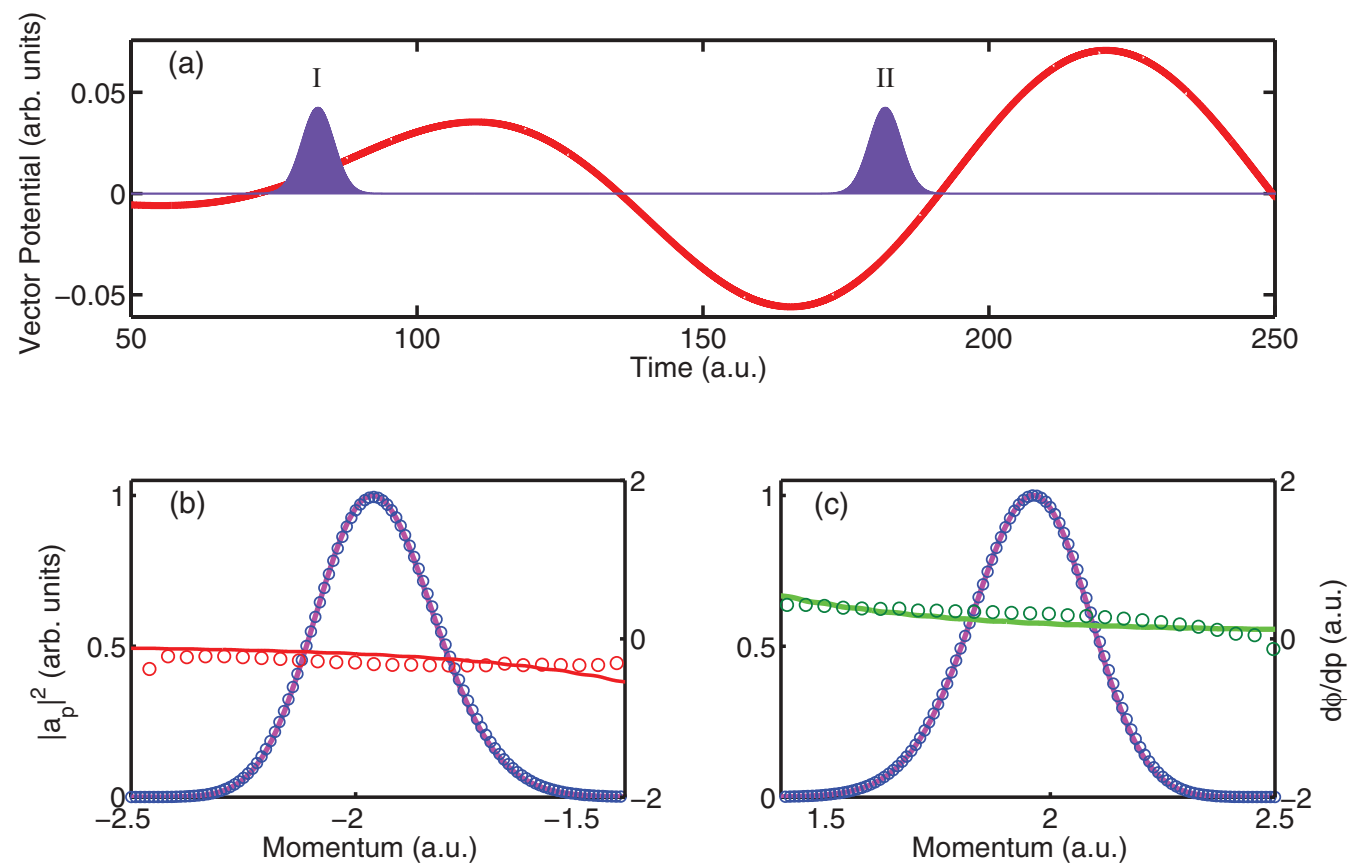

FIG. 5. (Color online) QSPIDER retrieval for the ground state of the helium model ion based on TDSE calculations. Lines and symbols are as defined in Fig. 4.

\section{CONCLUSION}

We have presented the QSPIDER technique to fully characterize the EWP and the complex dipole matrix element in an atom, ion, or molecule undergoing photoionization. The technique requires interaction of the system with a train of two attosecond pulses in the presence of a streaking IR field. QSPIDER can retrieve amplitudes and phases faithfully in a region defined by the central frequency and spectral width of the attosecond pulses. If the pulses are well known and the Coulomb-laser coupling is taken into account, the technique measures the complex dipole matrix element, which can be used in the future to access structural information of larger systems.

QSPIDER inherits most of the known issues of the SPIDER techniques. High resolution is needed to measure the interferogram. In the case of QSPIDER this concerns the energy or momentum resolution in the spectrometer. Several methods have been proposed to overcome these issues for SPIDER which may be helpful also in QSPIDER [34,35].

Since the phase is closely related to the Wigner time, QSPIDER will provide an alternative way to determine the delay in photoionization, which has previously been investigated with the streaking method [4].

\section{ACKNOWLEDGMENTS}

We are grateful to Jost Henkel for the insightful discussion and useful suggestion. This research was funded by the Spanish Ministerio de Ciencia e Innovación (MICINN), through Consolider Program SAUUL CSD2007-00013 and Research Project FIS2009-09522. Support from the Centro de Láseres Pulsados (CLPU) is also acknowledged. A.C. thanks the Secretaría Nacional de Ciencia Innovación y Tecnología (SENACYT) Panama for financial support. C.R. thanks the program Ramón y Cajal.
[1] P. Emma, R. Akre, J. Arthur et al., Nat. Photonics 4, 641 (2010).

[2] Z. Huang and K.-J. Kim, Phys. Rev. STAB 10, 034801 (2007).

[3] F. Krausz and M. Ivanov, Rev. Mod. Phys. 81, 163 (2009).

[4] M. Schultze, M. Fieß, N. Karpowicz et al., Science 328, 1658 (2010).

[5] K. Klünder, J. M. Dahlström, M. Gisselbrecht et al., Phys. Rev. Lett. 106, 143002 (2011).

[6] F. Quéré, J. Itatani, G. L. Yudin, and P. B. Corkum, Phys. Rev. Lett. 90, 073902 (2003); E. M. Kosik, L. Corner, A. S. Wyatt et al., J. Mod. Opt. 52, 361 (2005); J. Itatani, F. Quéré, G. L.
Yudin, M. Yu. Ivanov, F. Krausz, and P. B. Corkum, Phys. Rev. Lett. 88, 173903 (2002).

[7] T. Remetter, P. Johnsson, J. Mauritsson et al., Nat. Phys. 2, 323 (2006).

[8] J. Itatani, J. Levesque, D. Zeidler et al., Nat. Phys. 432, 867 (2004).

[9] S. Haessler, J. Caillat, W. Boutu et al., Nat. Phys. 6, 200 (2010).

[10] E. P. Wigner, Phys. Rev. 98, 145 (1955); C. A. A. De Carvalho and H. M. Nussenzveigb, Phys. Rep. 364, 83 (2002). 
[11] Y. Mairesse and F. Quéré, Phys. Rev. A 71, 011401(R) (2005).

[12] C. Iaconis and I. A. Walmsley, Opt. Lett. 23, 792 (1998).

[13] M. E. Anderson, L. E. E. Araujo, E. M. Kosik, and I. A. Walmsley, Appl. Phys. B 70, S85 (2000).

[14] M. Takeda, H. Ina, and S. Kobayashi, J. Opt. Soc. Am 72, 156 (1982).

[15] Few-Cycle Laser Pulse Generation and Its Applications, edited by F. X. Kärtner (Springer, Berlin, 2004), Vol. 95.

[16] M. E. Anderson, A. Monmayrant, S.-P. Gorza et al., Laser Phys. Lett. 5, 259 (2008).

[17] L. V. Keldysh, Zh. Eksp. Teor. Fiz. 47, 1945 (1964) [Sov. Phys. JETP 20, 1307 (1965)]; F. H. M. Faisal, J. Phys. B 6, L89 (1973); H. R. Reiss, Phys. Rev. A 22, 1786 (1980).

[18] D. B. Milošević, G. G. Paulus, D. Bauer et al., J. Phys. B 39, R203 (2006).

[19] Y. Mairesse, A. de Bohan, L. J. Frasinski et al., Science 302, 1540 (2003).

[20] T. Witting, D. R. Austin, and I. A. Walmsley, Opt. Lett. 34, 881 (2009).

[21] R. Thomas, Ph.D. thesis, Sweden, 2008.

[22] O. Smirnova, A. S. Mouritzen, S. Patchkovskii, and M. Y. Ivanov, J. Phys. B 40, F197 (2007); O. Smirnova, M. Spanner, and M. Ivanov, ibid. 39, S307 (2006).
[23] M. Ivanov and O. Smirnova, Phys. Rev. Lett. 107, 213605 (2011).

[24] J. Mauritsson, P. Johnsson, E. Gustafsson, A. L'Huillier, K. J. Schafer, and M. B. Gaarde, Phys. Rev. Lett. 97, 013001 (2006).

[25] U. Graf, M. Fieß, M. Schultze et al., Opt. Express 16, 18956 (2008).

[26] J. A. Pérez-Hernández, L. Plaja, and L. Roso, Opt. Express 17, 9891 (2009).

[27] W. Gropp and E. Lusk, The Message Passing Interface (MPI) Standard http://www.mcs.anl.gov/research/projects/mpi/.

[28] J. Sanders and E. Kandrot, CUDA by Example, An Introduction to General-Purpose GPU Programming (Addison-Wesley, Michigan, 2010).

[29] V. S. Yakovlev, J. Gagnon, N. Karpowicz, and F. Krausz, Phys. Rev. Lett. 105, 073001 (2010).

[30] J. W. Cooper, Phys. Rev. 128, 681 (1962).

[31] C. Ruiz, QFISHBOWL http://code.google.com/p/qfishbowl/.

[32] M. Frigo and S. G. Johnson, FFTw http://www.fftw.org/.

[33] E. V. van der Zwan and M. Lein, Phys. Rev. Lett. 108, 043004 (2012).

[34] D. R. Austin, T. Witting, and I. A. Walmsley, J. Opt. Soc. Am. B 26, 1818 (2009); Opt. Lett. 35, 1971 (2010).

[35] J. R. Birge, R. Ell, and F. X. Kärtner, Opt. Lett. 31, 2063 (2006). 